\title{
Fuzzy cell mapping on dynamical systems
}

\author{
Y. Song ${ }^{1}$, D. Edwards ${ }^{2}$ and V. S. Manoranjan ${ }^{3 *}$ \\ ${ }^{1}$ Savannah College of Art and Design, P. O. Box 3146, Savannah, GA 31402-3146, USA. \\ ${ }^{2}$ Department of Mechanical Engineering, University of Idaho, Moscow, ID 83844-0902, USA. \\ ${ }^{3}$ Department of Mathematics, Washington State University, Pullman, WA 99164-3113, USA.
}

Accepted 19 April, 2013

\begin{abstract}
Fuzzy cell mapping is a novel computational technique that combines fuzzy logic and a simple cell mapping method. In a simple cell mapping method, the information about mapping locations of image cells is never incorporated into the method. This limits the usage of a simple cell mapping method. In our fuzzy cell mapping method, we account for the mapping locations of image cells and incorporate the information by employing triangular membership functions. This paper demonstrates the use of fuzzy cell mapping on nonlinear dynamical systems. Our results indicate that fuzzy cell mapping can give good estimates on the global behavior of autonomous dynamical systems that posses limit cycles and strange attractors.
\end{abstract}

Key words: Fuzzy cell mapping, nonlinear dynamical systems, limit cycle, strange attractor.

\section{INTRODUCTION}

Nonlinear systems appear in many scientific disciplines such as engineering, physics, chemistry, biology, economics, and demography. Therefore, methods of analysis of nonlinear systems, which can provide a good understanding of their behavior, have wide applications. Although there are several analytical methods (Hsu, 1987), determining the global behavior of strongly nonlinear systems is still a substantially difficult task. The direct approach of numerical integration is a viable method. However, such an approach is sometimes prohibitively time consuming even with the powerful present-day computers.

In an attempt to find more efficient and practical ways of determining the global behavior of strongly nonlinear systems, methods such as cell-to-cell mapping were proposed and developed in the 1970's and 1980's (Hsu, 1987; Lind and Marcus, 1995). The basic idea behind the cell-to-cell mapping method is to consider the state space not as a continuum but rather as a collection of a large number of state cells, with each cell taken as a state entity. In the past, only two types of cell mappings, that is, simple cell mapping (SCM) and generalized cell mapping (GCM), have been investigated. A few years ago, Edwards and Choi (1997) proposed a new type of cell mapping method, namely fuzzy cell mapping (FCM). This method combines fuzzy logic and cell mapping techniques in a way to maintain the simplicity of SCM and achieve the results of GCM. Edwards and Choi (1997) were able to demonstrate the usefulness of their method by applying it to the Duffing equation, a nonlinear dynamical system where the time variable appears explicitly (non-autonomous system). In this paper, we extend the work of Edwards and Choi (1997) and show FCM's versatility by applying the FCM to various autonomous systems (nonlinear dynamical systems where the time variable does not appear explicitly) with simple steady states, limit cycles and strange attractors. Frequently, many real world mechanical, biological and

*Corresponding author. E-mail: mano@wsu.edu. 


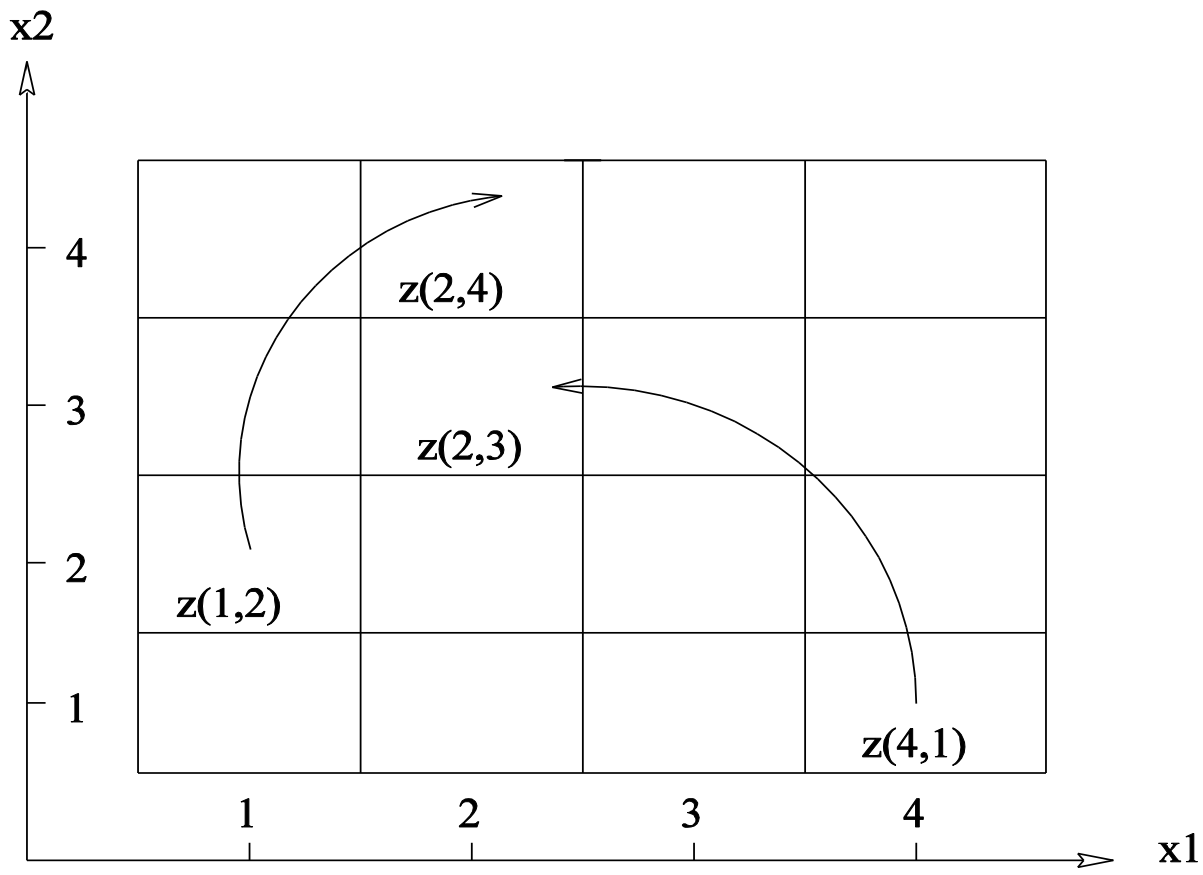

Figure 1. Cell structure and cell mapping illustration in two dimensions.

geometrical problems are formulated in terms of nonlinear autonomous dynamical systems (Jordan and Smith, 1989; Manoranjan et al., 2006; Manoranjan et al., 2008). So, there is an increasing need to consider and study methods that can provide a better understanding of such nonlinear dynamical systems.

Next are brief reviews on the different cell mapping methods; thereafter results of our study with FCM are presented.

\section{SIMPLE CELL MAPPING AND GENERALIZED CELL MAPPING}

In a cell-to-cell mapping, as mentioned before, the state space is considered as a collection of state cells, with each cell taken as a state entity. There are many ways to construct a cell structure over a given Euclidean state space. The simplest way is to construct a cell structure consisting of rectangular parallelepipeds of uniform size. Let $x_{i}, i=1,2, \ldots, N$ be the state variables of the state space. Let the coordinate axis of a state variable $x_{i}$ be divided into a large number, say $m$, of intervals of uniform interval size $h_{i}$. The interval $z_{i}$ along the $x_{i}$-axis is defined to be one which contains all $x_{i}$ (Figure 1) satisfying:

$$
\left(z_{i}-\frac{1}{2}\right) h_{i} \leq x_{i}<\left(z_{i}+\frac{1}{2}\right) h_{i}
$$

Here, by definition $z_{i}$ is an integer, such that $z_{i}=1,2, \ldots$, m. An $N$-tuple $\left(z_{1}, z_{2}, \ldots, z_{N}\right)$ is then called a cell vector and is denoted by $z$. A point $x$ with components $x_{i}$ belongs to a cell $z$ with components $z_{i}$ if and only if $x_{i}$ and $z_{i}$ satisfy Equation (1) for all $i$.

Practically, for all physical problems, only a finite region of a cell state space is of interest to us. Thus, all the cells outside of this finite region can be lumped together into a single cell, a sink cell, which will be assumed to map into itself in the mapping scheme. With this definition of the sink cell, one can assume $m$ is finite, as is the total number of cells, $m^{N}$.

Let $n$ represent the state of the system at $t=n T$, where $T$ is the sampling time interval, which can be of any duration, but is usually associated with the period of the forcing function or the natural period of the system. The transition for a cell mapping method from the state at $\xi(n)$ to the state at $\xi(n+1)$ can be represented in a matrix notation as:

$\xi(n+1)=C \xi(n)$

where, $C$ is the transition matrix of dimensions $m^{N} \times m^{N}$ and $\xi$ is a $m^{N}$-tuple obtained by rearranging the indices of the cell vector $z$ into one dimension such that $\xi_{k}$, the $k$ th component of $\xi$, represents the $k$ th cell for $k=1,2$, $\ldots, m^{N}$.

In SCM, the system of equations is integrated for the 


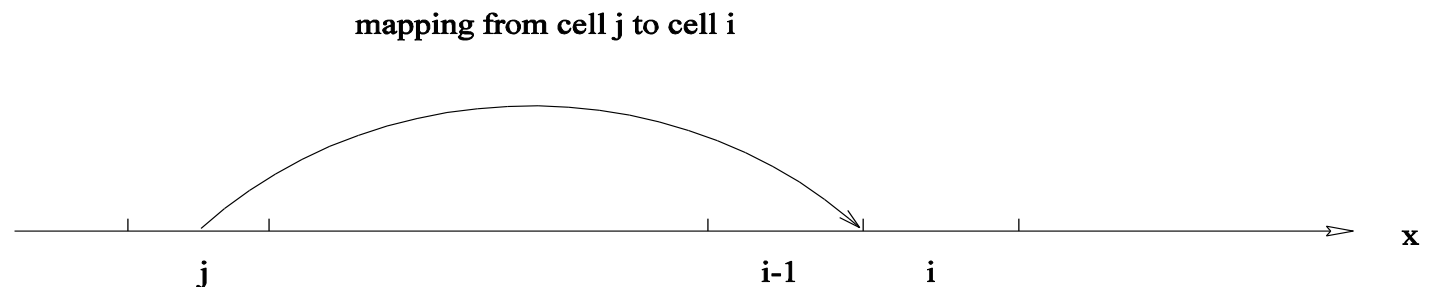

Figure 2. SCM in one dimension.

fixed time period $T$ using the center point of each cell as the initial condition. After the integration, each cell (represented by the center point of the cell), called the original cell or the domain cell, is mapped into another cell, the image cell (regardless of the actual image location in the cell). Mapping (2) becomes $\xi(n+1)=C_{S} \xi(n)$, where, the elements $c_{i j}(n)$ in the transition matrix $C_{S}$ are defined as the mapping from cell $j$ at $t=n T$ to cell $i$ at $t=(n+1) T$. The subscript on $C_{S}$ denotes SCM.

The matrix defines a Markov chain since only one element of each column is nonzero and which is equal to one. Though such a mapping, which results in a type of Poincare map, usually provides a good approximation to the global behavior of most systems, one cannot expect the method to disclose any structural details of the system behavior at a scale which is comparable to the cell size, as for a chaotic system, since only one point within the cell is used. One way to improve the power of the cell mapping method is to incorporate more system dynamics into the mapping. This leads to the generalized cell mapping method.

In GCM, the original cell is divided into subcells and each subcell is mapped, in the same fashion as in SCM, into an image cell. The probability that the original cell will map into an image cell is then based on where each subcell maps. Here, $\xi(n)$, instead of representing the cell vector as in SCM, represents the cell probability vector. The transition matrix becomes the transition probability matrix $C_{G}$. The elements, $c_{i j}(n)$, in $C_{G}$ map the probability that the state is in cell $j$ at $t=n T$ to the probability that the state will be in cell $i$ at $t=(n+1) T$.

Since each subcell represents a fixed, equal percentage of the original cell, the elements of a column in $C_{G}$ will sum to one with the number of nonzero elements being equal to the number of subcells. Once the probability matrix is determined, the iteration can be carried out by simple matrix-vector multiplications:

$\xi(n+k)=C_{G}^{k-1} \xi(n)$

This mapping process is also a Markov chain just like in SCM. The method is well suited, for instance, to find strange attractors of chaotic systems and the statistical properties associated with these systems. The principal drawback with this method is that it requires much more computations than the SCM method.

\section{FUZZY CELL MAPPING METHOD}

The GCM is needed because the SCM method does not provide an accurate estimate of the global properties for certain systems. This limitation exists because of the crisp way in which the mapping is allocated to the image cell. For example, in the one dimensional case, even when a mapping lands right in the middle of cell $i-1$ and cell $i$, where it is at equal distance from the center point of the two cells, by definition (1), cell $i$ is still the only image cell, though the mapping could equally belong to cell $i-1$ and cell $i$, (Figure 2). So, the SCM ignores an important piece of information, the actual mapping location.

In order to make use of all the information from SCM, FCM associates each cell with a membership function such that each cell, $\xi_{k}$, is described as a fuzzy set and the center point of the cell, $x_{\xi_{k}},\left(k=1,2, \ldots, m^{N}\right)$, as a fuzzy number. There are various ways to choose the membership functions. A simple, natural choice is:

$$
\begin{aligned}
& F_{\xi_{k}}(x)= \\
& \left\{\begin{array}{cc}
\prod_{i=1}^{i=N}\left(1-\frac{\left|x_{i}-\left(x_{\xi_{k}}\right)_{i}\right|}{h_{i}}\right) & \text { if }\left|x_{i}-\left(x_{\xi_{k}}\right)_{i}\right| \leq h_{i}, i=1,2, \ldots, N . \\
0 & \text { elsewhere }
\end{array}\right.
\end{aligned}
$$

Obviously, $F_{\xi_{k}}\left(x_{\xi_{k}}\right)=1$, and $F_{\xi_{k}}\left(x_{\xi_{j}}\right)=0$, for $k \neq j$, and $F_{\xi_{k}}(x)$ is symmetric about $x_{\xi_{k}}$ for all $k$. Intuitively, these are a set of triangular shaped functions in onedimension (Figure 3), and pyramid-like functions in twodimensions.

Let $x$ be the actual location of a mapping from an original cell. $\sum_{k=1}^{k=m^{N}} F_{\xi_{k}}(x)=1$, and the number of $\xi_{k}$ such that $F_{\xi_{k}}(x) \neq 0$ is at most $2^{N}$. 


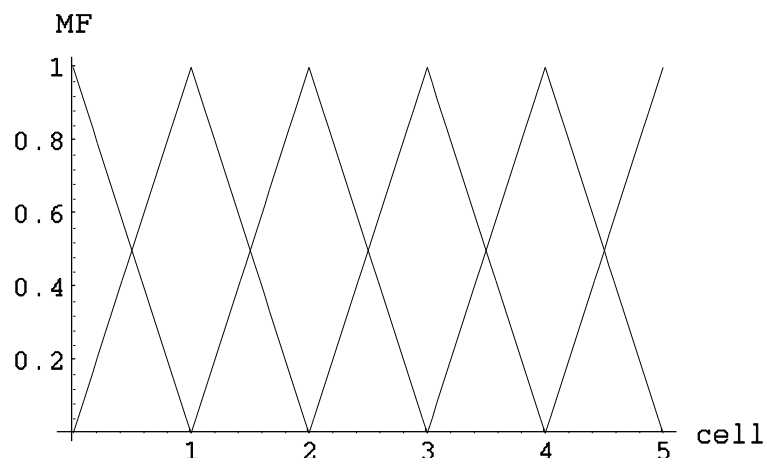

Figure 3. Triangular membership functions (MF) in one dimension.

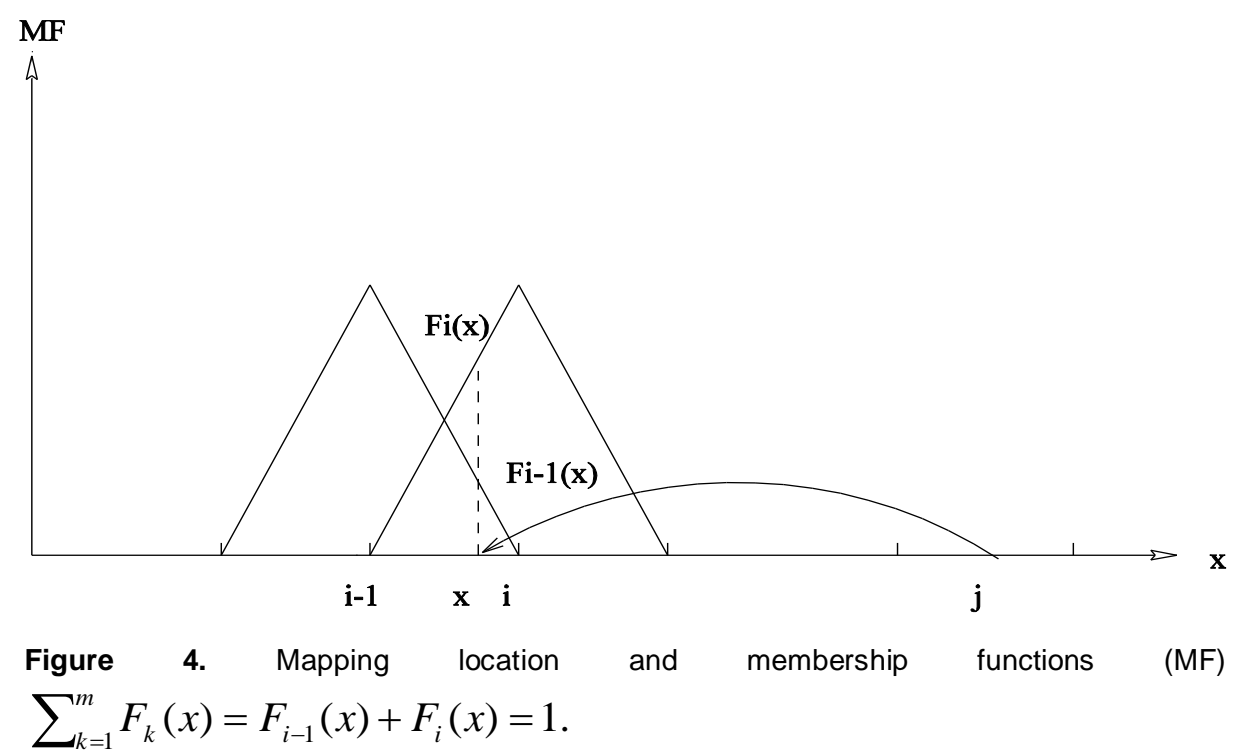

With such a choice of the membership functions, we define the transition matrix in (2) as $C_{F}$ such that the elements of $C_{F}, c_{i j}=F_{\xi_{i}}(x)$, where $x$ is the mapping location of the $j$-th cell (Figure 4).

So, FCM can be described by the following formula:

$$
\xi(n+k)=C_{F}^{k-1} \xi(n) .
$$

Again, the mapping process is a Markov chain.

The real advantage of FCM is that it uses only the information from SCM and no further integration is required.

\section{NUMERICAL EXPERIMENTS USING FCM}

We apply the FCM method on a variety of nonlinear dynamical systems. In the case of dynamical systems that possess either a limit cycle or a strange attractor, we solve the system using the FCM and Euler's methods. The results are compared pictorially.

\section{Use of FCM to determine stable equilibrium points}

Consider a two-species Lotka-Volterra competition model (Hofbauer and Sigmund, 1998),

$$
\begin{aligned}
& \dot{N}_{1}=N_{1}\left(1-N_{1}-2 N_{2}\right) \\
& \dot{N}_{2}=2 N_{2}\left(1-N_{2}-1.5 N_{1}\right)
\end{aligned}
$$

Here, $N_{1}$ and $N_{2}$ are the respective population densities of the competing species. This system has four equilibria, $(0,0),(0.5,0.25),(1,0)$, and $(0,1)$. The equilibria $(0,0)$ 


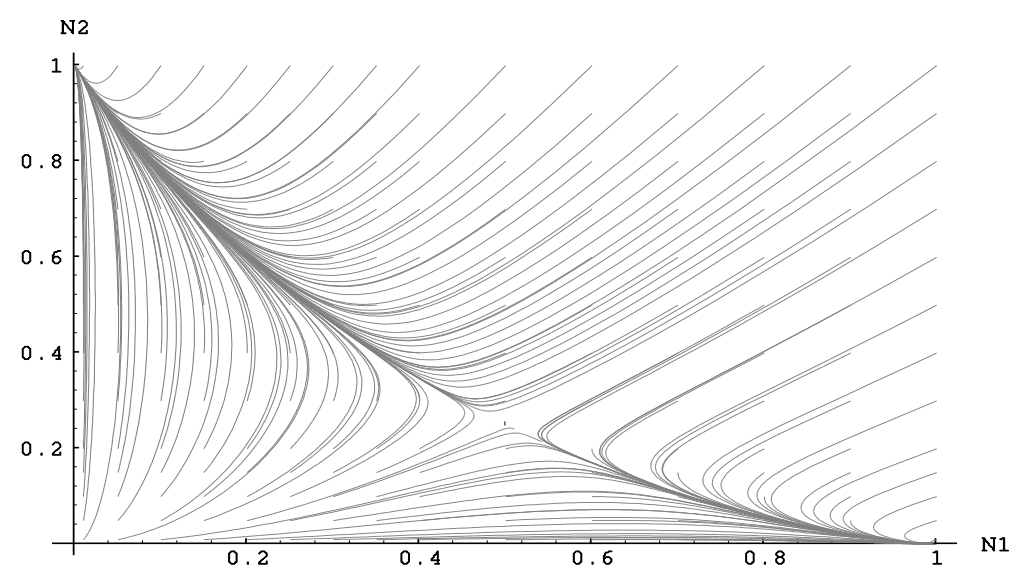

Figure 5. Phase trajectories for the competition model (6).

Table 1. Use of FCM on the competition model (6).

\begin{tabular}{cc}
\hline FCM converged to & Percentage of points that converged \\
\hline$(0,0)$ & 0.01 \\
$(0,1)$ & 69.85 \\
$(0.5,0.25)$ & 0.15 \\
$(1,0)$ & 29.99 \\
\hline
\end{tabular}

and $(0.5,0.25)$ are unstable; whereas, $(1,0)$ and $(0,1)$ are stable states with each having a domain of attraction separated by a separatrix that passes through $(0.5,0.25)$, the saddle point (Figure 5).

Table 1 presents the results obtained in solving the problem using the FCM method. Here, we uniformly divide the unit square in the phase plane into $101 \times 101=$ 10201 cells, and the center points were taken as the initial conditions for the integration.

The system was integrated for a time period of $T=6$. The process stopped when $\epsilon=\|\xi(n+1)-\xi(n)\|_{2} \leq 10^{-3}$ and it took 7 iterations. The one point that converged to the unstable equilibrium $(0,0)$ is $(0,0)$ itself and the points that converged to $(0.5,0.25)$; the saddle point, started on the separatrix.

\section{Use of FCM to determine limit cycles}

Consider the van der Pol equation (Seydel, 1988):

$\ddot{u}-\lambda\left(1-u^{2}\right) \dot{u}+u=0$,

with $\lambda=0.5$. This equation originally arose as an idealization of a spontaneously oscillating, or self-excited, valve circuit. This has a nice property where regions of positive and negative damping are separated by a closed loop (or path) known as a limit cycle.

Figure 6 shows the limit cycle solution for the van der Pol equation (7) obtained numerically by the Euler's method, which integrated the system for a time period of $T=10^{3}$, and by the FCM. For the FCM, the phase plane is divided into $303 \times 303=91809$ uniform cells over the region

$$
\begin{aligned}
& -3 \leq u_{1} \leq 3 \\
& -3 \leq u_{2} \leq 3
\end{aligned}
$$

where, $u_{1}=u$, and $u_{2}=\dot{u}$. Here we choose $T=2 \pi$, the period of the limit cycle. The figure presents the limit cycle determined by the FCM after 3 iterations. The FCM solution and the Euler solution practically overlap on each other.

\section{FCM can be used to determine strange attractors for chaotic systems}

Edwards and Choi (1997) gave such an example which was the Duffing equation, a non-autonomous system. Here, we apply the FCM method to two different 


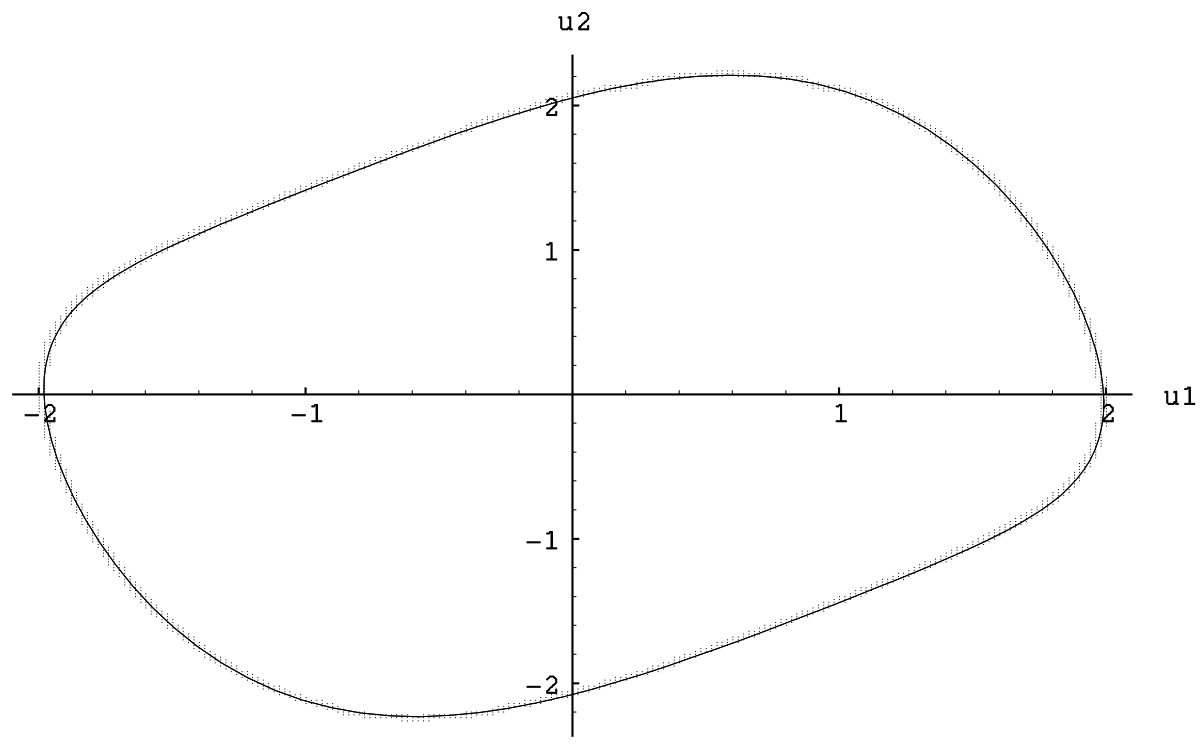

Figure 6. Limit cycle solution for the van der Pol Equation (7) (Solid line - Euler's method, Points - FCM).

nonlinear autonomous systems.

First we consider the Lorenz equations (Lorenz, 1963):

$$
\begin{aligned}
& \dot{x}_{1}=10\left(-x_{1}+x_{2}\right) \\
& \dot{x}_{2}=18 x_{1}-x_{2}-x_{1} x_{3} \\
& \dot{x}_{3}=-\frac{8}{3} x_{3}+x_{1} x_{2}
\end{aligned}
$$

These equations arose in a model for convective motion in the atmosphere. The system (9) is known to possess a chaotic (or strange) attractor which results in a bistable pole-reversing behavior that is aperiodic, even without any random input.

The strange attractor determined by the Euler's method, which integrated the system for a time period of $T=10^{4}$, is shown in Figure 7. Figure 8 presents the same attractor determined by the FCM. The region,

$$
\begin{array}{r}
-20 \leq x_{1} \leq 20, \\
-20 \leq x_{2} \leq 20, \\
0 \leq x_{3} \leq 40,
\end{array}
$$

in the phase space is divided into $61 \times 61 \times 61=226981$ cells. For $3 \times 3$ systems like this one, choosing the smallest cell size was limited by our computing resources. Here $T=10, \epsilon=10^{-5}$, and it took 85 iterations for the process to come to a stop.

We also applied the FCM to the chaotic system given in Beltrami (1987):

$$
\begin{aligned}
& \dot{x}_{1}=x_{1} x_{2}-\mu x_{1} \\
& \dot{x}_{2}=\left(x_{3}-\gamma\right) x_{1}-\mu x_{2} \\
& \dot{x}_{3}=1-x_{1} x_{2}
\end{aligned}
$$

with $\mu=2$ and $\gamma=5$. This system is known to model the magnetic field reversal of the earth and similar to Lorenz system possesses a strange attractor.

Figure 9 shows the strange attractor determined by the Euler's method with an integration period $T=10^{4}$. Figure 10 presents the strange attractor determined by the FCM. The region,

$$
\begin{aligned}
-8 & \leq x_{1} \leq 8, \\
-5 & \leq x_{2} \leq 5, \\
1 & \leq x_{3} \leq 10,
\end{aligned}
$$

in the phase space was considered with $60 \times 60 \times 60=216000$ cells.

Note that, if an initial point is taken on the $x_{3}$-axis, the solution will go out of the region defined by (12) and fall into the sink cell. Thus, $x_{3}$-axis is not in the domain of attraction. Therefore, one should pay special attention when constructing the cell structure to avoid such points being taken as centers of the cells.

\section{Conclusions}

From the above study, one can see that the FCM method 

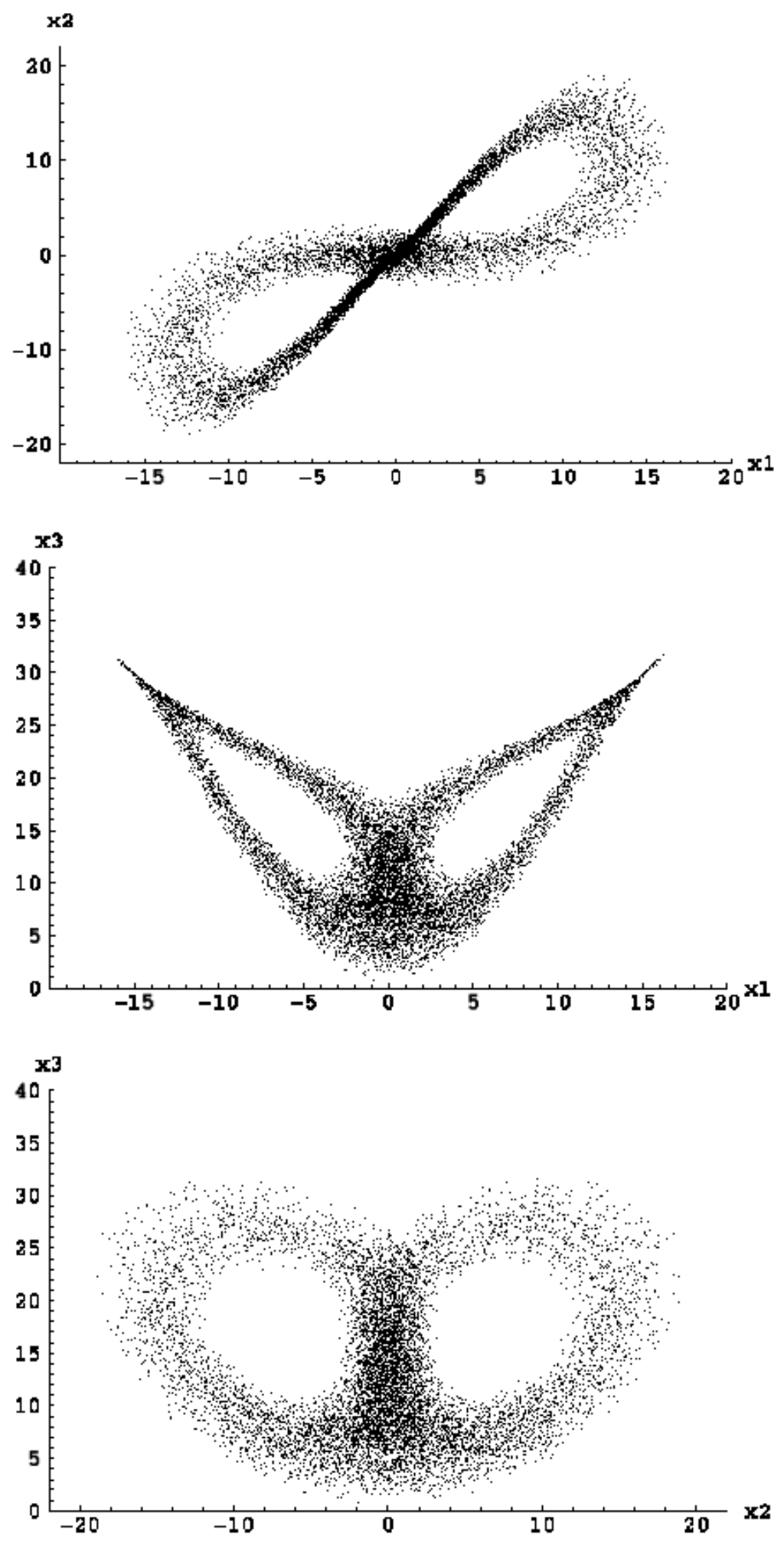

Figure 7. Strange attractor of the Lorenz equation (9) determined by the Euler's method $\left(T=10^{4}\right)$.

gives very good estimates on the global behavior for a variety of nonlinear dynamical systems. The results compare pretty well with that of Euler's method. We have demonstrated the versatility of FCM on autonomous 

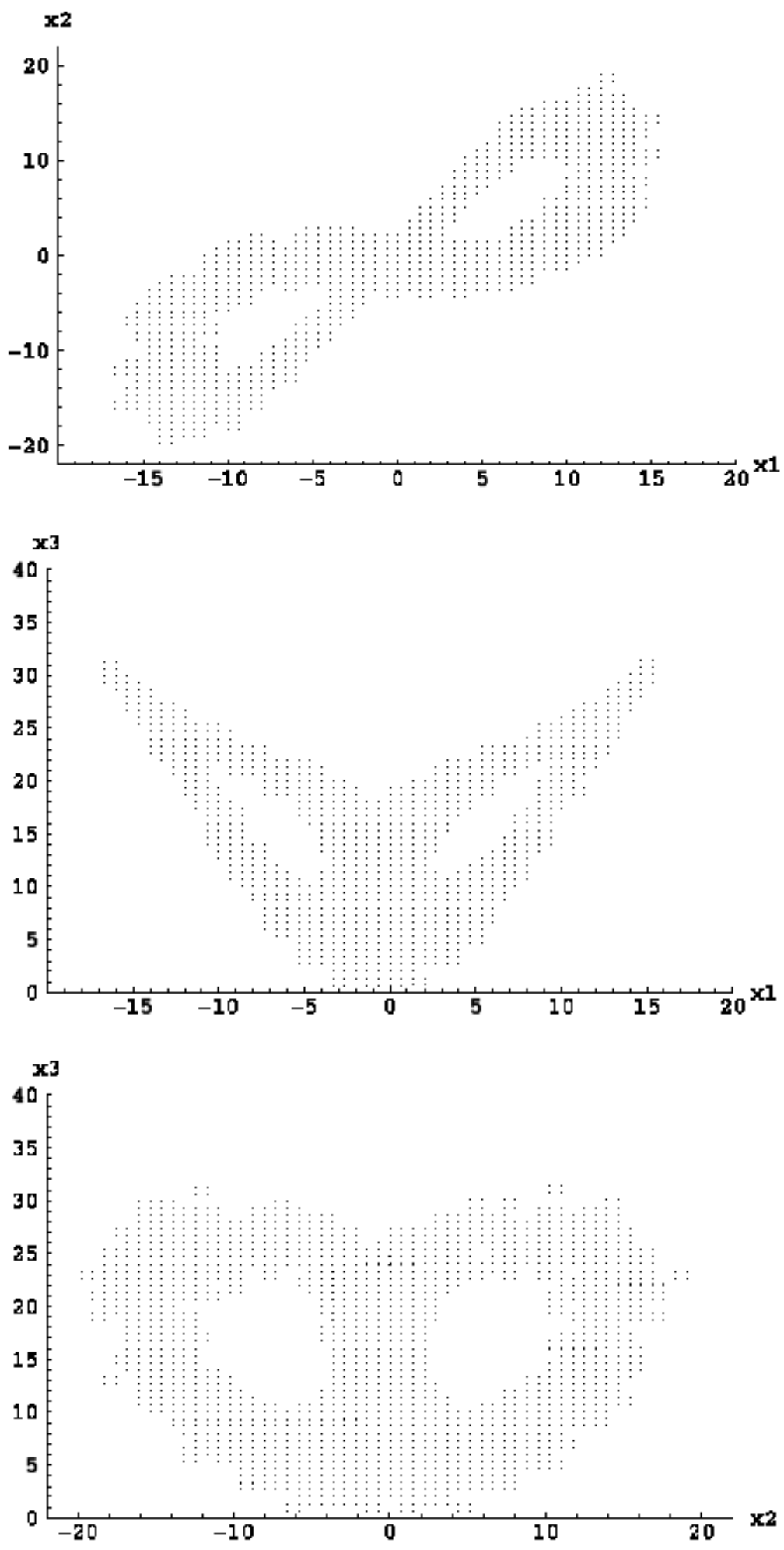

Figure 8. Strange attractor of the Lorenz equation (9) determined by the FCM $(61 \times 61 \times 61$ cells $)$.

systems with limit cycles and strange attractors. Best of all, the method is simple and straight forward. Therefore, the method is easy to understand and implement. One observation worth mentioning is that the FCM method 

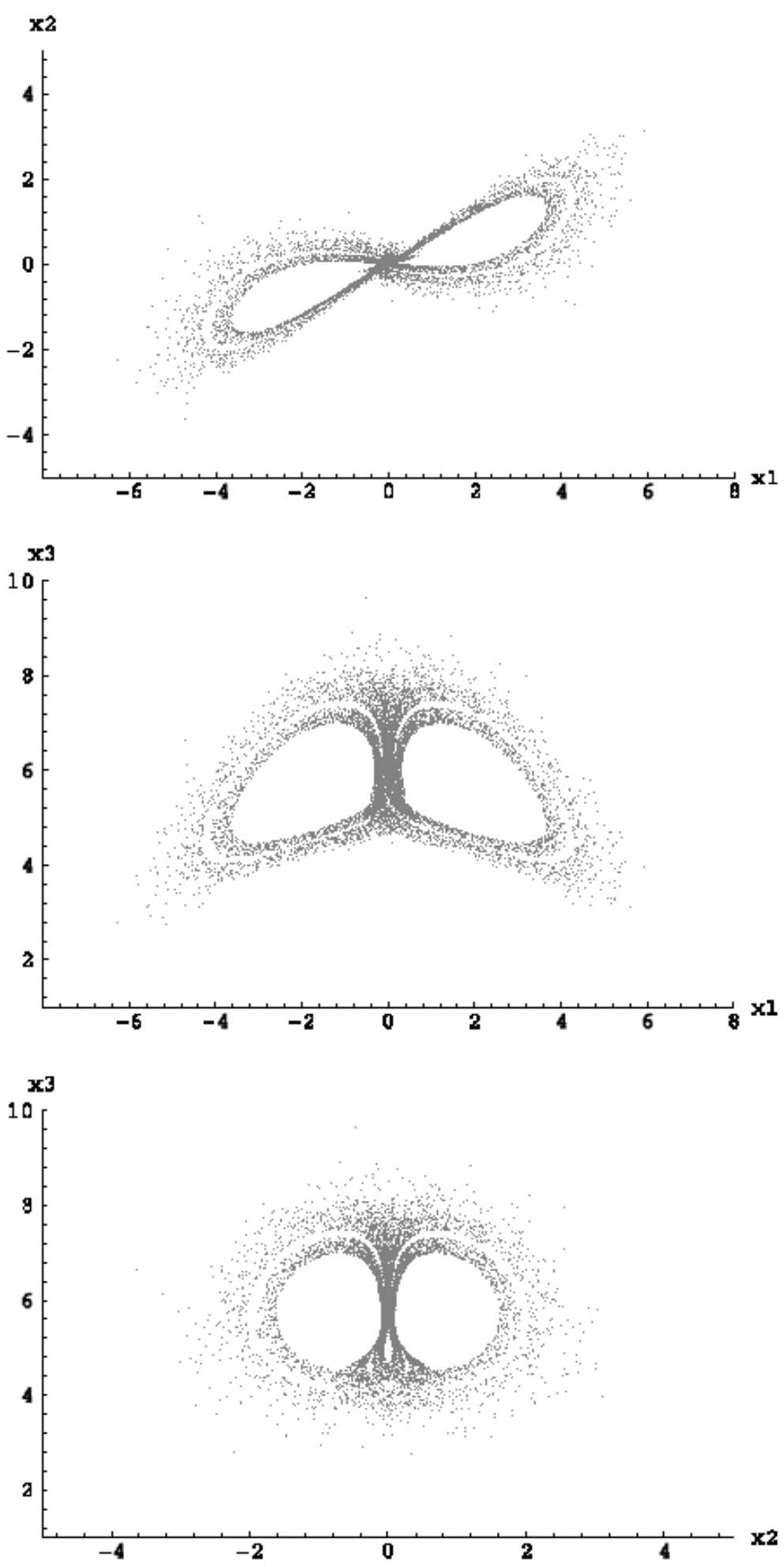

Figure 9. Strange attractor of the chaotic model (11), determined by the Euler's method $\left(T=10^{4}\right)$.

uses a very sparse matrix, $C_{F}$, although it is usually large. Actually only $2^{N}$ rows are nonzero in each column in a matrix of size $m^{N} \times m^{N}$, where $N$ is the dimension of the phase space and $m$ is the number of cells the 

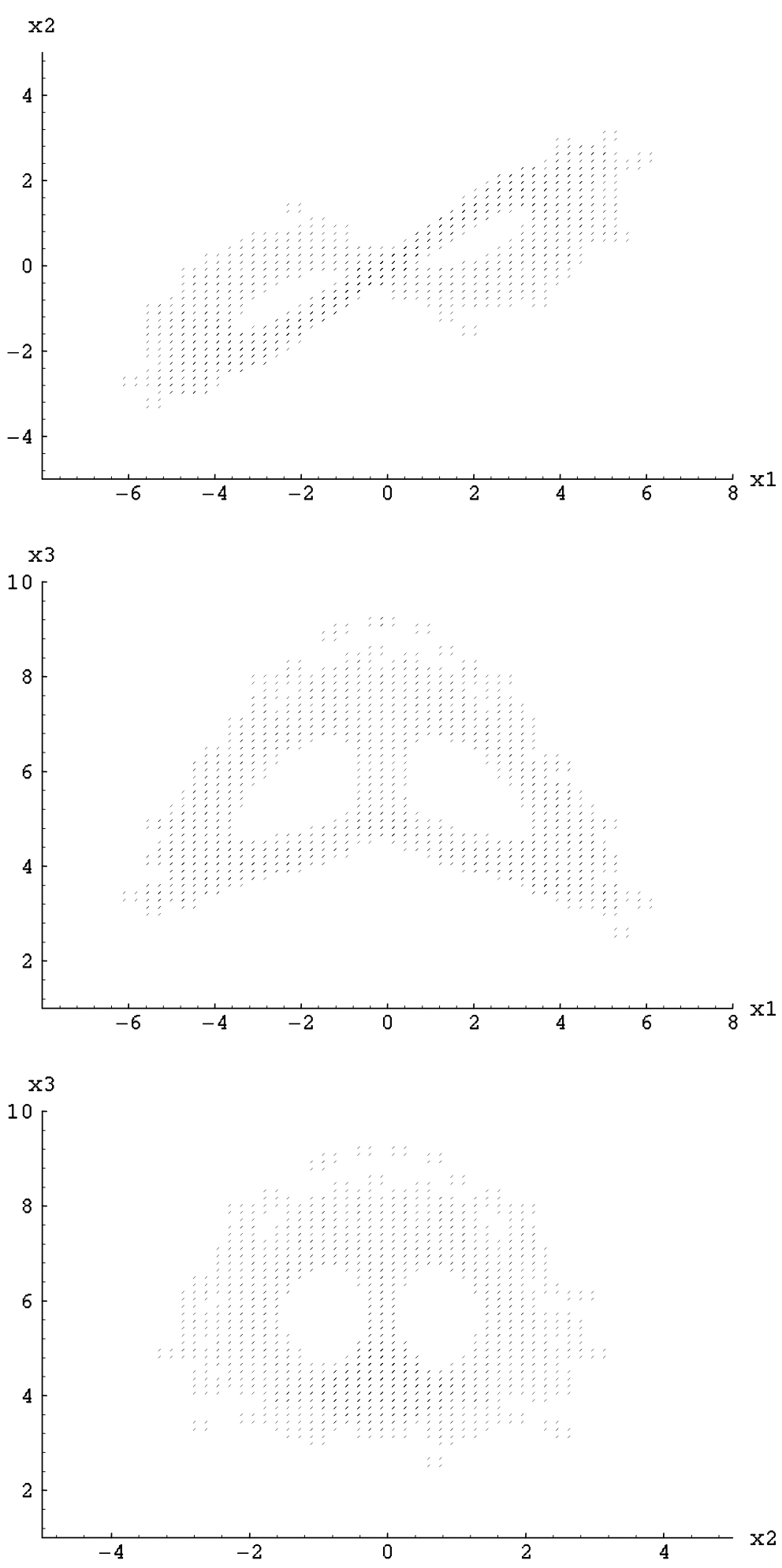

Figure 10. Strange attractor of the chaotic model (11), determined by the FCM ( $60 \times 60 \times 60$ cells).

phase space is divided into along each direction. When proper storage techniques are used, one can usually deal with quite large matrices.

The key for the FCM method is the construction of the 
membership functions. The reason for choosing the set of membership functions used here is that it is simple and it works. But it is by no means the only choice. The shortcoming for using such a generic membership function is that it ignores the details of the system and treats all kinds of systems the same.

One should be able to choose the membership functions based on the information from the system, such as the direction and the magnitude of the phase trajectory at each mapping location in the image cell. Tailoring the membership functions for the system should make the FCM method more efficient allowing the iterations to converge faster and currently we are exploring this approach.

The partition of the state space into cells can also be thought of as converting the nonlinear dynamical system into a symbolic dynamical system. However, not any partition results in a Markov partition. The work by Lind and Marcus (1995) presents a rigorous theory for Markov partitions and shows how topological partitions can be chosen so that a given nonlinear dynamical system can be faithfully represented by a symbolic dynamical system. Our study has demonstrated the power of fuzzification and how faithfully the fuzzy cell mapping method was able to produce the limiting behaviors of various nonlinear dynamical systems. In our future study, using the ideas presented in Lind and Marcus (1995), we intend to develop some theoretical results for the fuzzy cell mapping method.

\section{REFERENCES}

Beltrami E (1987). Mathematics for Dynamic Modeling. Academic Press: San Diego.

Edwards D, Choi HT (1997). Use of fuzzy logic to calculate the statistical properties of strange attractors in chaotic systems. Fuzzy Sets Syst. 88:205-217.
Hofbauer J, Sigmund K (1998). Evolutionary Games and Population Dynamics. Cam-bridge University Press: Cambridge.

Hsu CS (1987). Cell-to-cell mapping. Springer-Verlag: New York.

Jordan DW, Smith P (1989). Nonlinear Ordinary Differential Equations. Clarendon Press: Oxford.

Lind D, Marcus B (1995). An Introduction to Symbolic Dynamics and Coding. Cambridge University Press: Cambridge.

Lorenz EN (1963). Deterministic nonperiodic flow. J. Atmos. Sci. 20:130-141.

Manoranjan VS, Gomez MAO, Harwood RC (2008). Modelling Algae Self-Replenishment. J. Interdiscip. Math. 11:681-694.

Manoranjan VS, Rajapakse I, Krueger JM (2006). Oscillations in a Neuronal Assembly - A Phenomenological Model. Int. J. Comput. Appl. Math. 1:57-64.

Seydel R (1988). From Equilibrium to Chaos. Elsevier Science Publishing: New York. 